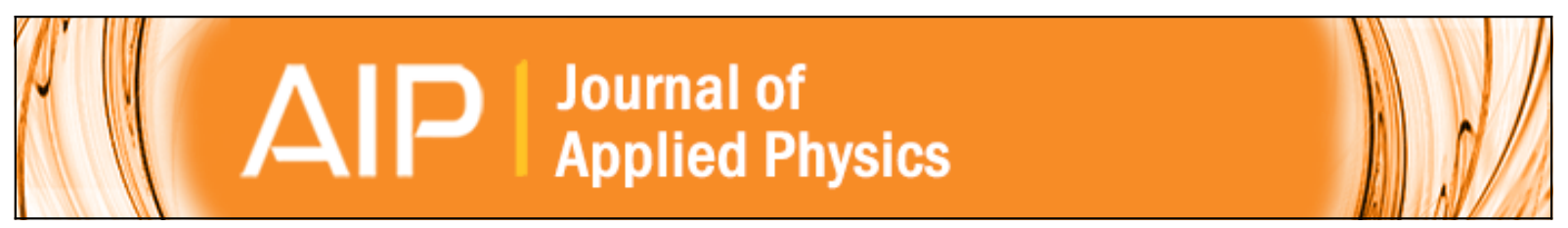

\title{
Electromagnetic power absorption due to bumps and trenches on flat surfaces
}

Carlos Pérez-Arancibia, Peng Zhang, Oscar P. Bruno, and Y. Y. Lau

Citation: Journal of Applied Physics 116, 124904 (2014); doi: 10.1063/1.4896361

View online: http://dx.doi.org/10.1063/1.4896361

View Table of Contents: http://scitation.aip.org/content/aip/journal/jap/116/12?ver=pdfcov

Published by the AIP Publishing

\section{Articles you may be interested in}

Electromagnetic absorption mechanisms in metal nanospheres: Bulk and surface effects in radiofrequencyterahertz heating of nanoparticles

J. Appl. Phys. 109, 124306 (2011); 10.1063/1.3600222

Enhanced absorption of metals over ultrabroad electromagnetic spectrum

Appl. Phys. Lett. 95, 121106 (2009); 10.1063/1.3227668

Effect Of Crystallization Annealing And Surface Oxidation On Electromagnetic Wave Absorption Behavior Of FeSiBNbCu Nanocrystalline Alloy

AIP Conf. Proc. 1003, 258 (2008); 10.1063/1.2928958

Electromagnetic absorption of a bimetallic spherical particle

Low Temp. Phys. 31, 587 (2005); 10.1063/1.2001639

Electromagnetic and absorption properties of some microwave absorbers

J. Appl. Phys. 92, 876 (2002); 10.1063/1.1489092

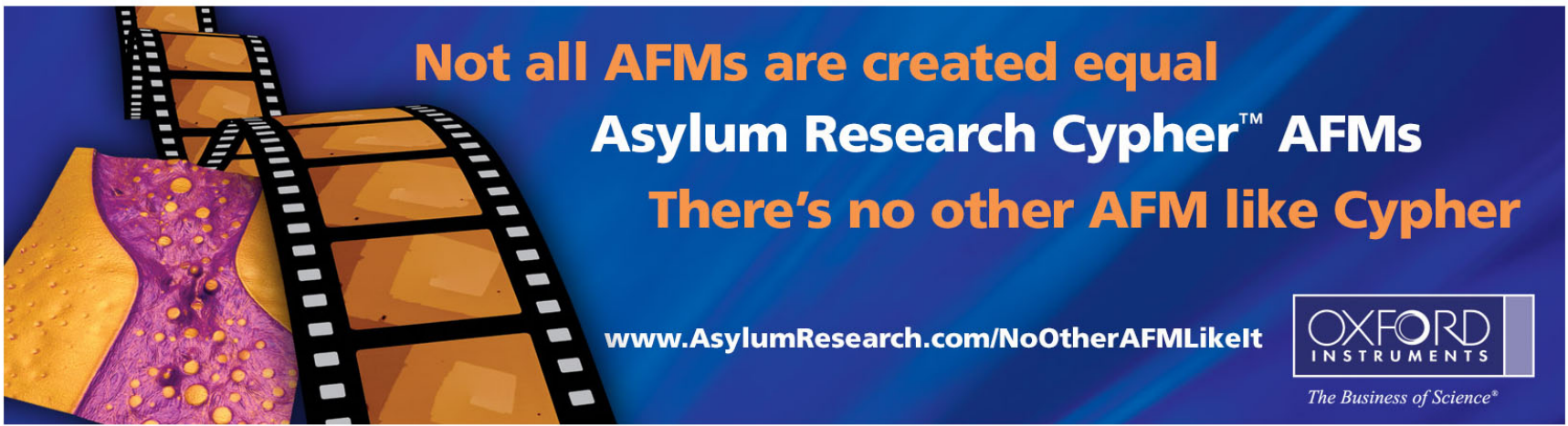




\title{
Electromagnetic power absorption due to bumps and trenches on flat surfaces
}

\author{
Carlos Pérez-Arancibia, ${ }^{1, a)}$ Peng Zhang, ${ }^{2, b)}$ Oscar P. Bruno, ${ }^{1, c)}$ and Y. Y. Lau ${ }^{2, d)}$ \\ ${ }^{1}$ Computing and Mathematical Sciences, California Institute of Technology, Pasadena, California 91125, USA \\ ${ }^{2}$ Department of Nuclear Engineering and Radiological Sciences, University of Michigan, Ann Arbor, \\ Michigan 48109, USA
}

(Received 14 July 2014; accepted 12 September 2014; published online 26 September 2014)

\begin{abstract}
This paper presents a study of the absorption of electromagnetic power that results from the interaction of electromagnetic waves and cylindrical bumps or trenches on flat conducting surfaces. Configurations are characterized by means of adequately selected dimensionless variables and parameters so that applicability to mathematically equivalent (but physically diverse) systems can be achieved easily. Electromagnetic fields and absorption increments caused by such surface defects are evaluated by means of a high-order integral equation method which resolves fine details of the field near the surface, and which was validated by fully analytical approaches in a range of computationally challenging cases. The computational method is also applied to problems concerning bumps and trenches on imperfect conducting planes for which analytical solutions are not available. Typically, we find that absorption is enhanced by the presence of the defects considered, although, interestingly, absorption can also be significantly reduced in some cases-such as, e.g., in the case of a trench on a conducting plane where the incident electric field is perpendicular to the plane. Additionally, it is observed that, for some small-skin-depths large-wavelengths, the absorption increment is proportional to the increase in surface area. Significant physical insight is obtained on the heating that results from various types of electromagnetic incident fields. (C) 2014 AIP Publishing LLC. [http://dx.doi.org/10.1063/1.4896361]
\end{abstract}

\section{INTRODUCTION}

Interaction of electromagnetic waves with rough surfaces is an important topic in science and engineering: surface roughness is known to cause excessive electromagnetic power absorption, ${ }^{1-6}$ and it is one of the major limiting factors to the performance of radio-frequency (RF) cavities and slow wave structures ${ }^{3,7-12}$ with an impact on communication systems, ${ }^{1,8,11}$ particle accelerators, ${ }^{1,9,13}$ and material characterization at microwave frequencies. ${ }^{14,15}$ The enhanced power loss due to surface and interface roughness exerts significant effects on signal integrity of microelectronic circuits. ${ }^{16-20}$ Other effects due to surface roughness, such as local electric and magnetic field enhancements, may trigger RF-breakdown ${ }^{5,8,11,19-23}$ and abrupt quenching ${ }^{2,7,9}$ (i.e., rapid loss of superconductivity) of a superconducting cavity.

Early work on characterization of the effects of surface roughness on electromagnetic power loss dates back to the 1940s when Morgan ${ }^{1}$ first studied the increased resistive power loss for a periodic two-dimensional (2D) ridged structure. The propagation of electromagnetic waves on rough surfaces was studied extensively by Wallis, Maradudin, and Mills in the 1970s, including uses of the Born approximation and approximations of first order in the surface roughness. ${ }^{24,25}$ In 1996, Groiss et al. ${ }^{26}$ obtained an empirical scaling relation by fitting solutions of boundary value problems

\footnotetext{
a)Electronic mail: cperezar@caltech.edu

b)Electronic mail: umpeng@umich.edu

${ }^{\mathrm{c})}$ Electronic mail: obruno@caltech.edu

${ }^{\mathrm{d})}$ Electronic mail: yylau@umich.edu
}

obtained by use of the finite-element method (FEM); this scaling relation was eventually adopted in the commercial FEM software High Frequency Structure Simulator $(\mathrm{HFSS})^{27}$ for characterization of conductor losses arising from surface roughness. Despite extensive usage, the frequency regime of validity for this scaling relation is not fully determined as yet. 5

Recently, both periodic ${ }^{28,29}$ and random $^{16}$ roughness profiles have been studied using various methods. Holloway and Kuester $^{28}$ calculated the power loss associated with periodic conducting and superconducting rough interfaces using a generalized impedance boundary condition. However, the fine details of the field near surfaces are not resolved. Matsushima and Nakata ${ }^{29}$ utilized the equivalent source method to numerically study periodic rectangular, triangular, and semielliptical grooves both transverse and parallel to current flow. There are also studies on modeling the surface roughness with randomly distributed bosses on surface. ${ }^{30,31}$ Accurate analytical scaling has been obtained for the power absorption due to a single hemispherical protrusion, of arbitrary permittivity $\varepsilon$, conductivity $\sigma$, and permeability $\mu$, located on a resonant cavity's surface. ${ }^{5}$

In this paper, we provide an accurate assessment of the enhanced electromagnetic power absorption caused by small local surface defects consisting of cylindrical bumps or trenches, where we assume the radius $a$ of the defect is much smaller than the free space wavelength $\lambda(a \ll \lambda)$ and the radius of curvature of the surface at the location of the defect (in the absence of the defect) is much larger than $\lambda$, so that the surface can be considered as locally flat in the absence of 
the defect. The 2D surface defect is assumed to be in the form of either a single semi-cylindrical bump or trench located on an otherwise flat surface (Figs. 1 and 3). The calculation is two-dimensional, using a high-order integral equation method (HIEM). ${ }^{32}$ Here, we focus on the long wavelength case, where the radius of the bump (or trench), denoted by $a>0$, is assumed to be smaller than the electromagnetic wavelength $\lambda>0$ exterior to the bump (or trench): $a / \lambda \ll 1$. The short wavelength limit, $a / \lambda>1$, together with some new features such as "pseudo resonances," will be addressed in a separate publication. Our use of HIEM instead of other methods such as the FDTD ${ }^{33}$ and the FEM $^{34}$ is driven by a number of motivations: (1) The HIEM can effectively produce valuable high-frequency results whereas at high frequencies FDTD and FEM become badly behaved; the HIEM does not require either (2) discretization of a large computational domain (only the trench or bump curve needs to be discretized and the effect of the planar dielectric interface is automatically incorporated via the dielectric-layer Green function) or (3) use of artificial boundary conditions (also called absorbing or transparent boundary conditions); and (4) the HIEM is highly accurate, even when used at relatively low discretization densities. In the present paper, we concentrate only on the validation of HIEM, and the new physical insights the highly accurate HIEM solutions provide in the low frequency regimes. As shown in Sec. II A, in particular, the solutions produced by the HIEM can be utilized to evaluate accurately and reliably the power absorption caused by surface defects.

To validate the numerical methodology while providing useful physical insight, in Sec. II B we consider problems whereby a semi-cylindrical bump lies on a perfectly conducting surface (PEC) under various electromagnetic incident fields (Figs. 1 and 2; Zhang et al., ${ }^{5}$ Pérez-Arancibia and Bruno $^{32}$ ). Thus validated, the HIEM approach is used to treat important structures which defy analytical treatment, namely, cylindrical bumps and trenches on finitely conducting flat surfaces (Fig. 3) - where, letting $\sigma$ denote the conductivity at the RF frequency $\omega$, the permittivity in the bump equals the (complex, scalar) permittivity $\varepsilon_{1}=\varepsilon_{1 r}+i \sigma / \omega$ in the pristine conducting half-space. The permittivity $\varepsilon_{2}$ in the region outside the conducting structure is taken as a real constant, and the magnetic permeability $\mu=\mu_{1}=\mu_{2}$ is assumed real and constant throughout space. Clearly, letting $\delta=\left(2 / \omega \mu_{1} \sigma\right)^{1 / 2}$ denote the skin depth associated with the conducting material, the ratio $\delta / a$ may take on an arbitrary value ranging from zero to infinity: $\delta \rightarrow \infty$ corresponds to an insulating surface and $\delta=0$ corresponds to a perfectly conducting surface.

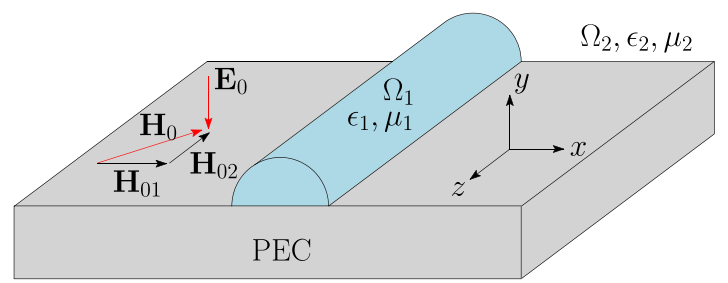

FIG. 1. A small semi-cylindrical bump on a perfectly conducting surface whose local wave electric field is $\mathbf{E}_{0}$ and local wave magnetic field $\mathbf{H}_{0}$ in the absence of the bump.

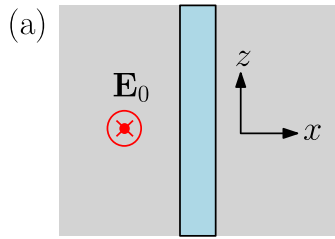

(b)

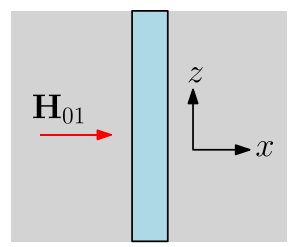

(c)

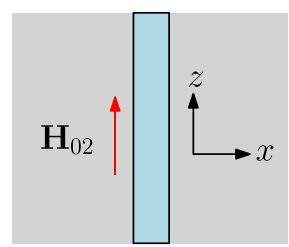

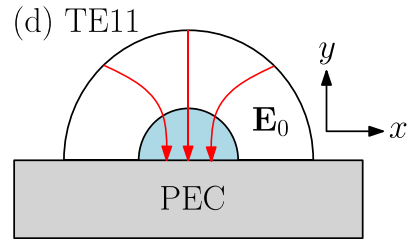

(e) TM01

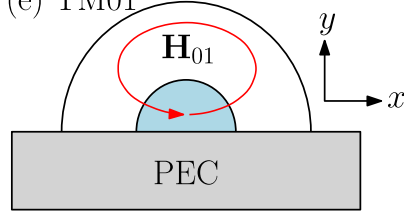

(f) TE01

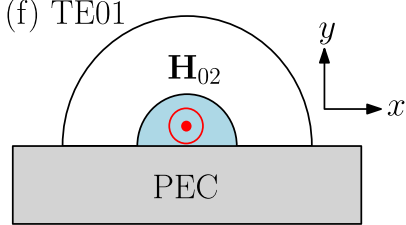

FIG. 2. Incident fields considered, following Ref. 5, for a bump on a PEC plane shown in Fig. 1. An incident electromagnetic field is either given by an electric field (a) $\mathbf{E}_{0}$ perpendicular to the PEC plane and associated magnetic field; by a magnetic field (b) $\mathbf{H}_{01}$ parallel to the plane and perpendicular to the bump axis and associated electric field; or by a magnetic field (c) $\mathbf{H}_{02}$ parallel to the bump axis and associated electric field. According to Ref. 5 , these incident fields are approximated by (semi-)cylindrical eigenmodes, namely the (d) TE11 mode, (e) TM01 mode, and (f) TE01 mode, respectively, which can themselves be expressed in closed-form in terms of products of Bessel functions and complex exponentials.

Figures 7-12, which display heating profiles that arise for a wide range of standing-wave and plane-wave RF incident fields, demonstrate the great variety of surface heating profiles that may arise as a result of the surface defects under consideration. Section III discusses the physical mechanisms that give rise to such variety of heating patterns; in all, our HIEM model gives rise to significant physical insight on the heating induced under incident fields of various types.

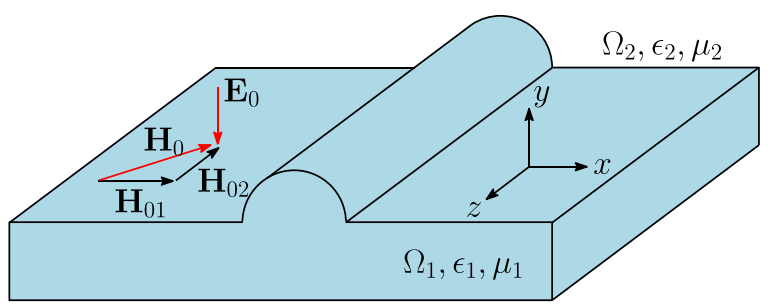

(a) Bump.

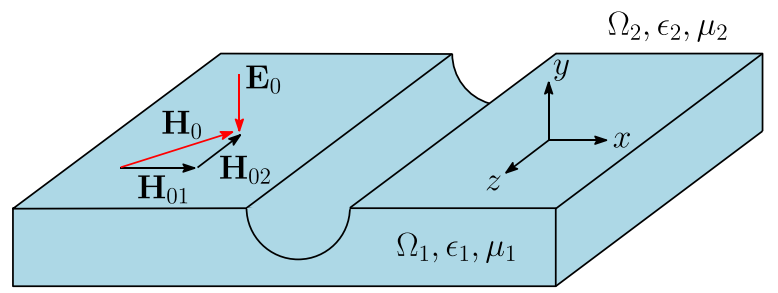

(b) Trench.

FIG. 3. A semi-cylindrical (a) bump, and (b) trench, on a conducting surface of finite conductivity. The bump is made of the same material as the flat surface. 
The remainder of this paper is organized as follows: Section II describes the physical problems we consider as well as corresponding analytical solutions based on ideas presented in Zhang et al. ${ }^{5}$ Section III presents our main results on enhanced power absorption due to surface defects. Concluding remarks, finally, are provided in Sec. IV.

\section{NUMERICAL METHOD AND BENCHMARKS}

\section{A. Electromagnetic theory and high-order integral solver}

In this section, we consider the problem of scattering of a general plane or standing electromagnetic wave by a cylindrical defect (bump or trench) on a conducting or perfectly conducting half-plane (Figs. 1 and 3). For clarity, in what follows we call $\Omega_{1}$ the region occupied by the conducting material, and we let $\Omega_{2}$ denote the region outside $\Omega_{1}$. The symmetry of the defects allows for the total electromagnetic field to be expressed as a linear combination of transverse electric (TE) and transverse magnetic (TM) fields. As is well known, ${ }^{35}$ in TE-polarization the total electromagnetic field is given by $(\mathbf{E}, \mathbf{H})=\left(-\left(i \omega \varepsilon_{j}\right)^{-1} \nabla \times\left(u_{j} \mathbf{e}_{3}\right), u_{j} \mathbf{e}_{3}\right)$ in $\Omega_{j}, j=1$, 2 , where the $z$-component of the total magnetic field $u_{j}$ satisfies the Helmholtz equation ${ }^{35}$

$$
\nabla^{2} u_{j}+k_{j}^{2} u_{j}=0 \quad \text { in } \quad \Omega_{j}, \quad j=1,2,
$$

with wavenumber $\quad k_{j}=\omega \sqrt{\varepsilon_{j} \mu_{j}}\left(0 \leq \arg k_{j}<\pi / 2\right)$. Similarly, in TM-polarization the total electromagnetic field is given by $(\mathbf{E}, \mathbf{H})=\left(u_{j} \mathbf{e}_{3},\left(i \omega \mu_{j}\right)^{-1} \nabla \times\left(u_{j} \mathbf{e}_{3}\right)\right)$ in $\Omega_{j}, j=1$, 2 , where the $z$-component of the electric field $u_{j}$ satisfies the Helmholtz equation (1).

The continuity of the tangential components of $\mathbf{E}$ and $\mathbf{H}$ at the interface between two conducting/dielectric media ${ }^{35}$ (CD) under TE and TM polarizations leads, respectively, to the transmission conditions

$$
\begin{aligned}
\frac{1}{\varepsilon_{1}} \frac{\partial u_{1}}{\partial \mathbf{n}}=\frac{1}{\varepsilon_{2}} \frac{\partial u_{2}}{\partial \mathbf{n}}, \quad u_{1}=u_{2} \quad \text { on } \quad \Gamma_{12}(\mathrm{TE}-\mathrm{CD}), \\
\frac{1}{\mu_{1}} \frac{\partial u_{1}}{\partial \mathbf{n}}=\frac{1}{\mu_{2}} \frac{\partial u_{2}}{\partial \mathbf{n}}, \quad u_{1}=u_{2} \quad \text { on } \quad \Gamma_{12}(\mathrm{TM}-\mathrm{CD}) .
\end{aligned}
$$

Here $\mathbf{n}$ denotes the unit normal to the boundary $\Gamma_{12}$ between $\Omega_{1}$ and $\Omega_{2}$. On a PEC surface, on the other hand, the tangential components of the electric field vanish, ${ }^{35}$ and we thus have

$$
\begin{aligned}
& \frac{\partial u_{j}}{\partial \mathbf{n}}=0, \quad(\text { TE-PEC }), \\
& u_{j}=0, \quad(\text { TM-PEC } .
\end{aligned}
$$

The Helmholtz equation (1) and associated boundary conditions (2) lead to a well-posed 2D scattering problem from which the unknown functions $u_{j}$ in $\Omega_{j}(j=1,2)$ can be obtained. The problems of scattering thereby formulated can be solved numerically by utilizing the HIEM introduced in Ref. 32. The HIEM is based on single-layer-potential field representations, efficient evaluation of Sommerfeld integrals, and quadrature rules which produce the single-layer potential with high-order accuracy in spite of Green-function and geometric singularities. In detail, according to the nomenclature established in Ref. 32, the conducting bump on the PEC plane (Fig. 1) on one hand, and both the bump and the trench on the finitely conducing plane (Fig. 3) on the other, correspond to problems of types III and I, respectively.

In this paper, we consider the power dissipation that results from three different types of incident electromagnetic fields for which (i) the electric field $\mathbf{E}_{0}$ is perpendicular to the conducting surface with wavevector $\mathbf{k}$ perpendicular to the cylinder axis (see Fig. 3), where the complex amplitude of the electric field equal to $\left(\mathrm{e}^{i k_{2} x}+\mathrm{e}^{-i k_{2} x}\right) / 2=\cos \left(k_{2} x\right)$; (ii) the magnetic field $\mathbf{H}$ (denoted in this case by $\mathbf{H}_{01}$ ) is perpendicular to axis of the defect with wavevector $\mathbf{k}$ perpendicular to the planar interface, where the complex amplitude of the incident magnetic field is equal to $\mathrm{e}^{-i k_{2} y}$; and (iii) the magnetic field $\mathbf{H}$ (denoted in this case by $\mathbf{H}_{02}$ ) is parallel to the conducting surface and to the axis of the defect, where the complex amplitude of the magnetic field equal to $\left(\mathrm{e}^{i k_{2} x}+\mathrm{e}^{-i k_{2} x}\right) / 2=\cos \left(k_{2} x\right)$. In what follows we present analytical expressions for the power dissipation for each one of these cases.

The incident fields in cases (i) and (iii) clearly lead to TE problems, and thus, to scalar functions $u_{j}(j=1,2)$ which satisfy Eqs. (1) and (2a), and which, for the case of the bump on the PEC plane, additionally satisfy Eq. (2c). Once the functions $u_{j}$ have been obtained by application of the HIEM, the average electromagnetic power dissipated inside an arbitrary conducting region $D \subset \Omega_{1}$ is given by ${ }^{35}$

$$
\begin{aligned}
P & =\int_{D} \sigma|\mathbf{E}|^{2} \mathrm{~d} x=\frac{\sigma}{\omega^{2}\left|\varepsilon_{1}\right|^{2}} \int_{D}\left|\nabla u_{1}\right|^{2} \mathrm{~d} x \\
& =\frac{\sigma}{\omega^{2}\left|\varepsilon_{1}\right|^{2}}\left[\int_{\partial D} \bar{u}_{1} \frac{\partial u_{1}}{\partial \mathbf{n}} \mathrm{d} s+k_{1}^{2} \int_{D}\left|u_{1}\right|^{2} \mathrm{~d} x\right],
\end{aligned}
$$

where the last expression in Eq. (3) results from the previous one by integration by parts. The HIEM provides both the magnetic field $u_{1}$ in $\Omega_{1}$ and its normal derivative on the boundary of the defect, and thus, Eq. (3) allows us to evaluate the overall absorption without need to produce the gradient of $u_{1}$. (Since in the HIEM the function $u_{j}$ is represented by means of a single-layer potential, direct evaluation of the gradient by differentiation of the corresponding integral representation leads to integral operators with kernels that possess strong singularities at the boundary of the defect and which are therefore difficult to integrate. The last expression in (3) allows us to bypass this difficulty).

The incident-field in case (ii), in turn, leads a TM problem, and thus, to scalar functions $u_{j}$ which satisfy Eqs. (1) and (2b), and which, for the case of the bump on the PEC plane, additionally satisfy Eq. (2d). In this case, the power dissipated inside an arbitrary conducting region $D \subset \Omega_{1}$ can be produced by means of the expression

$$
P=\int_{D} \sigma|\mathbf{E}|^{2} \mathrm{~d} x=\int_{D} \sigma\left|u_{1}\right|^{2} \mathrm{~d} x .
$$

\section{B. Analytical model for code validation}

The problem of electromagnetic power absorption by a cylindrical bump on a perfectly conducting surface can be 
solved analytically in the asymptotic limit $a / \lambda \ll 1$, without any restriction on the skin depth $\delta$ (cf., e.g., Ref. 5). (In fact solutions based on separation of variables can also be produced for general values of $a / \lambda$; cf., e.g., Ref. 32.) These analytical results are used for code validation in what follows.

Following Zhang et al., ${ }^{5}$ for the incident fields determined by $\mathbf{E}_{0}, \mathbf{H}_{01}$, and $\mathbf{H}_{02}$ on a semi-cylindrical bump (Figs. 2(a)-2(c)), we now consider the auxiliary problems described in Figs. 2(d)-2(f). Figures 2(d)-2(f) show a perfectly conducting semi-cylindrical cavity (of infinite axial length) of radius $b_{E_{0}}, b_{H_{01}}, b_{H_{02}}$, whose natural frequency for the fundamental TE11, TM01, and TE01 mode is $\omega$ when this cavity is empty (i.e., by setting $b_{E_{0}}=1.84118 / \omega \sqrt{\varepsilon_{2} \mu_{2}}$, $\left.b_{H_{01}}=b_{H_{02}}=3.83171 / \omega \sqrt{\varepsilon_{2} \mu_{2}}\right)$, and whose vacuum eigenmode at the center of cavity is $\mathbf{E}_{0}, \mathbf{H}_{01}$, and $\mathbf{H}_{02}$, respectively, as shown in Figs. 2(d)-2(f). We now insert a semicylindrical bump of radius $a>0$, complex permittivity $\varepsilon_{1}=\varepsilon_{1 r}+i \sigma / \omega$, and real permeability $\mu_{1}$ at the center of this semi-cylindrical cavity (Figs. 2(d)-2(f)). The high degree of symmetry (the fields configuration inside the perfect conducting semi-cylindrical cavity are equivalent to those of a cylindrical cavity of the same radius) allows us to analytically calculate the perturbation on the eigenmode and the perturbation on the eigenfrequency by this semi-cylindrical $\operatorname{rod}^{5,36,37}$ (see the Appendix).

The damping rates of the eigenmodes, $\gamma$, are related to the average power loss $P$ through the general relationship, ${ }^{38}$ $P=2 \gamma U$, where $U$ is the average electromagnetic energy stored in the eigenmode of the empty cavity. The power dissipation per unit axial length for the electromagnetic fields associated with the electric field $\mathbf{E}_{0}$ and the magnetic fields $\mathbf{H}_{01}$ and $\mathbf{H}_{02}$ in Figs. 2(a)-2(c), or equivalently in Figs. 2(d)-2(f), denoted by $P_{E_{0}}, P_{H_{01}}$, and $P_{H_{02}}$, respectively, then reads

$$
\begin{gathered}
P_{E_{0}}=2 \gamma_{E_{0}} U_{E_{0}}, \\
U_{E_{0}}=0.4774 \times\left(\frac{1}{2} \varepsilon_{2}\left|E_{0}\right|^{2}\right) \frac{\pi b_{E_{0}}^{2}}{2}, \\
P_{H_{01}}=2 \gamma_{H_{01}} U_{H_{01}}, \\
U_{H_{01}}=0.4774 \times\left(\frac{1}{2} \mu_{2}\left|H_{01}\right|^{2}\right) \frac{\pi b_{H_{01}}^{2}}{2}, \\
P_{H_{02}}=2 \gamma_{H_{02}} U_{H_{02}}, \\
U_{H_{02}}=0.1622 \times\left(\frac{1}{2} \mu_{2}\left|H_{02}\right|^{2}\right) \frac{\pi b_{H_{02}}^{2}}{2},
\end{gathered}
$$

where $\gamma_{E_{01}}, \gamma_{H_{01}}$, and $\gamma_{H_{02}}$ are given in Eqs. (A2), (A5), and (A8), respectively, where $E_{0}\left(H_{01}, H_{02}\right)$ is the peak value of the RF electric (magnetic) field of the TE11 (TM01, TE01) mode at the center of the semi-cylindrical cavity in the absence of the bump (Fig. 2), and $b_{E_{0}}\left(b_{H_{01}}, b_{H_{02}}\right)$ is the radius of the cavity for the TE11 (TM01, TE01) mode (cf. Eqs. (A1), (A4), and (A7)).

Figure 4 shows $\gamma_{E_{0}, H_{01}, H_{02}}$ (Eqs. (A2), (A5), and (A8)), and their asymptotes (Eqs. (A3), (A6), and (A9)), in terms of $(\lambda / a)^{2}$, as a function of $\delta / a$ for a number of values $\lambda / a$, for the special case $\mu_{1}=\mu_{2}=\mu_{0}$, and $\varepsilon_{1 r}=\varepsilon_{2}=\varepsilon_{0}$. The values of $P$

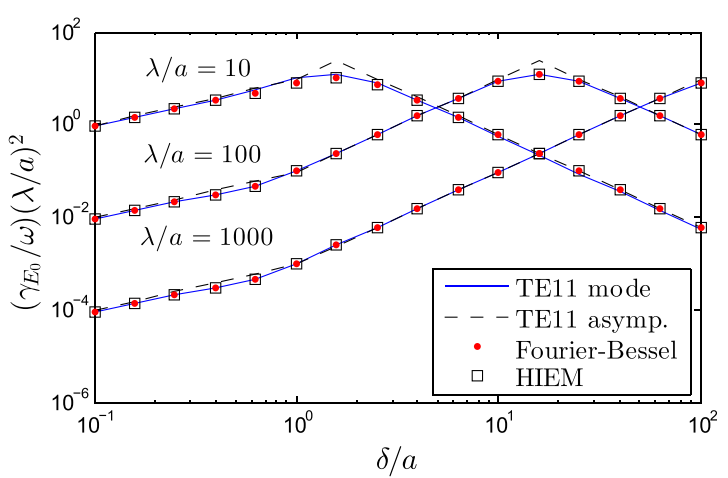

(a) $\mathbf{E}_{0}$

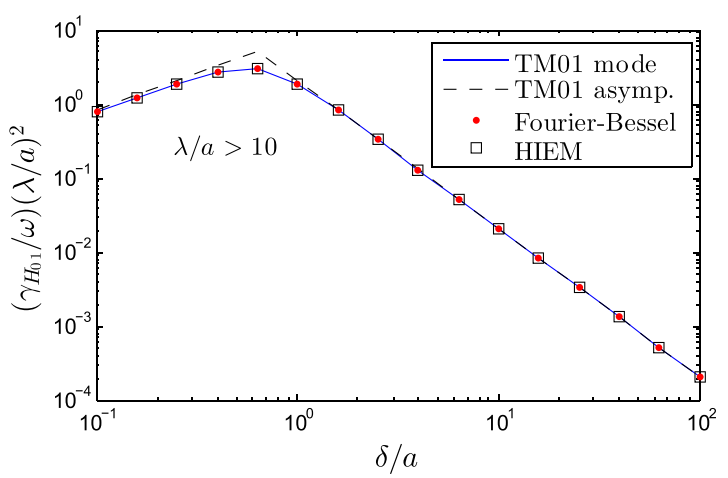

(b) $\mathbf{H}_{01}$

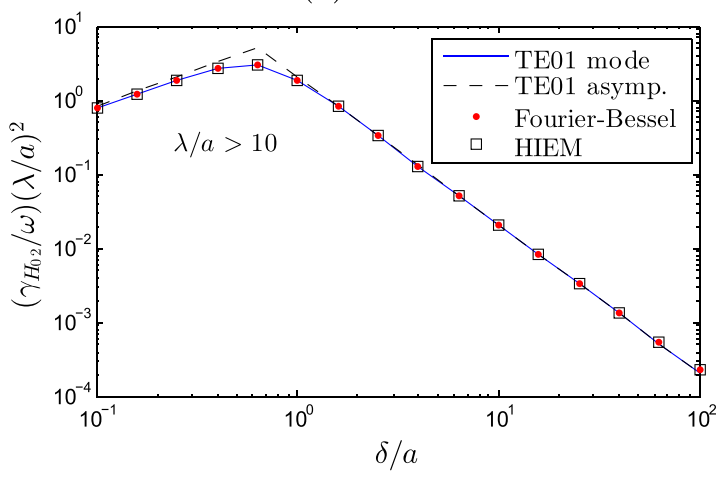

(c) $\mathbf{H}_{02}$

FIG. 4. (a) $\gamma_{E_{0}}$ (Eq. (A2)) and its asymptotes (Eq. (A3)), (b) $\gamma_{H_{01}}$ (Eq. (A5)) and its asymptotes (Eq. (A6)), and (c) $\gamma_{H_{02}}$ (Eq. (A8)) and its asymptotes (Eq. (A9)), in terms $(\lambda / a)^{2}$, as a function of $\delta / a$ for a number of values of $\lambda / a$, for the special case $\mu_{1}=\mu_{2}=\mu_{0}$ and $\varepsilon_{1 r}=\varepsilon_{2}=\varepsilon_{0}$. Red dots represent the exact value computed using the Fourier-Bessel expansion ${ }^{32}$ of the exact solution of the problem of scattering, and the black squares represent the values obtained using HIEM.

(see Eqs. (3) and (4)) obtained from the Fourier-Bessel expansion of the exact solution of the problem of scattering (see the Appendix in Refs. 32 and 39) and numerical calculation from HIEM are superimposed to the curves in Fig. 4. Almost identical results are obtained. This may be considered as a validation of HIEM code. The latter will be carried on to characterize the additional power absorption for surface roughness on a surface of finite conductivity (Sec. III).

It is interesting to note that $\gamma_{H_{01}} / \omega$ and $\gamma_{H_{02}} / \omega$ give identical values, for $\lambda / a \gg 1$, shown in Figs. 4(b) and 4(c). Also note from Eqs. (A6) and (A9) that these asymptotes, after normalizing to $(a / \lambda)^{2}$, are independent of $\lambda / a$. From the intersection of the asymptotic expressions, one estimates that the 
maximum value of $\left(\gamma_{E_{0}} / \omega\right)(\lambda / a)^{2}$ is about 30 , occurring at a value of $\delta / a=(1 / 2 \pi)(\lambda / a)$, as shown in Fig. 4(a); whereas both the maximum values of $\left(\gamma_{H_{01}} / \omega\right)(\lambda / a)^{2}$ and $\left(\gamma_{H_{02}} / \omega\right)$ $(\lambda / a)^{2}$ are about 5 , occurring at a value of $\delta / a=0.63$ approximately, as shown in Figs. 4(b) and 4(c). Taking the ratio of Eqs. (A3) and (A6) (or Eq. (A9)), one obtains $\gamma_{H_{01}, H_{02}} / \gamma_{E_{0}}=$ $0.034(\lambda / a)^{2} \gg 1$ for a good conducting bump $(\delta / a \ll 1)$, as is also evident in Fig. 4. Thus, heating by the RF magnetic field dominates over the heating by the RF electric field when $\delta / a<1 ., 36,37,40$ We emphasize that, physically, "heating by the RF magnetic field" is actually the same as ohmic heating due to the induced RF electric field within the bump by the external RF magnetic field component, and this is the physical basis of derivation of the magnetic polarizability for nonmagnetic materials by Landau and Lifshitz. ${ }^{41}$

\section{RESULTS AND DISCUSSION}

Since no analytical models have been found (to the authors' knowledge) for the problem of a cylindrical bump or a trench on a flat surface with finite conductivity (Fig. 3), we perform numerical calculations using HIEM only. Following the code validation test of a bump on a perfectly conducting surface in Sec. II B, here we still assume that the incident electromagnetic fields may be considered as linear combination of three cases: $\mathbf{E}_{0}, \mathbf{H}_{01}$, and $\mathbf{H}_{02}$, each oscillating at the same frequency $\omega$ (Fig. 3). As described in Sec. II A, the case $\mathbf{E}_{0}$ is calculated with a standing wave, which is formed by sending two TEM waves (both with electric field perpendicular to the flat surface) of equal amplitude and frequency from both sides of the bump, with the phases adjusted so that the total electric field (which is perpendicular to the flat surface) is maximum at the center of the bump when the bump is absent, (total magnetic field is zero there). The case $\mathbf{H}_{02}$ is calculated similarly with the same standing wave, but with the phases of the TEM waves adjusted so that the total magnetic field (which is along the defect axis) is maximum at the center of the bump when it is absent (total electric field is zero there). The case $\mathbf{H}_{01}$ is calculated by sending a TEM wave propagating perpendicularly to the surface, with the magnetic field perpendicular to the axis of the defect, where a standing wave is formed by the incident and reflected waves. Since the surface is assumed to be conductive, the electric field on the surface is small and the magnetic field (perpendicular to the bump axis) dominates near the surface. For simplicity, in this section we only consider cases in which the dielectric constant of the bump and the flat surface coincide.

For each of the three cases $\mathbf{E}_{0}, \mathbf{H}_{01}$, and $\mathbf{H}_{02}$, we compute the power absorption per unit axial length, $P_{\text {flat }}$, due to the flat surface (when the defect is absent) using formula (3) or (4) depending on the polarization, integrating in the domain $D=D_{\text {flat }}=[-2 a, 2 a] \times[0,-4 a]$. The boundary of the flat conducting plane is taken to be $y=0$. Similarly, the power absorption per unit axial length, $P_{\text {rough }}$, due to the rough surface (when the defect is present) is computed using formula (3) or (4) integrating in the domain $D=D_{\text {rough }}=D_{\text {flat }} \cup B$ in the case of the bump, and $D=D_{\text {rough }}=D_{\text {flat }} \backslash B$ in the case of the trench, where $B=\left\{(x, y) \in \mathbb{R}^{2}: \sqrt{x^{2}+y^{2}}<a\right\}$. All the required integrals in Eqs. (3) and (4) are computed with high-order accuracy by means of a combination of Clenshaw-Curtis quadratures in polar and Cartesian coordinates. Figures 5(a)-5(c) (Figs. 6(a)-6(c)) plot the ratio of the enhanced power absorption due to the bump (trench) to the ohmic loss of the pristine flat surface

$$
\frac{\Delta P}{P_{\text {flat }}}=\frac{P_{\text {rough }}-P_{\text {flat }}}{P_{\text {flat }}}=\frac{P_{\text {rough }}}{P_{\text {flat }}}-1,
$$

as a function of $\delta / a$, for various values of $\lambda / a$, and for the three incident fields under consideration.

Figures 7, 8, and 9 (Figs. 10, 11, and 12) display in great detail the magnitude of the electric field near the surface of the finitely conducting surface with the bump (trench) for all the three different cases $\mathbf{E}_{0}, \mathbf{H}_{01}$ and $\mathbf{H}_{02}$ respectively. The color scale in all these plots is adjusted so that the maximum (red) and the minimum (blue) match the corresponding maximum

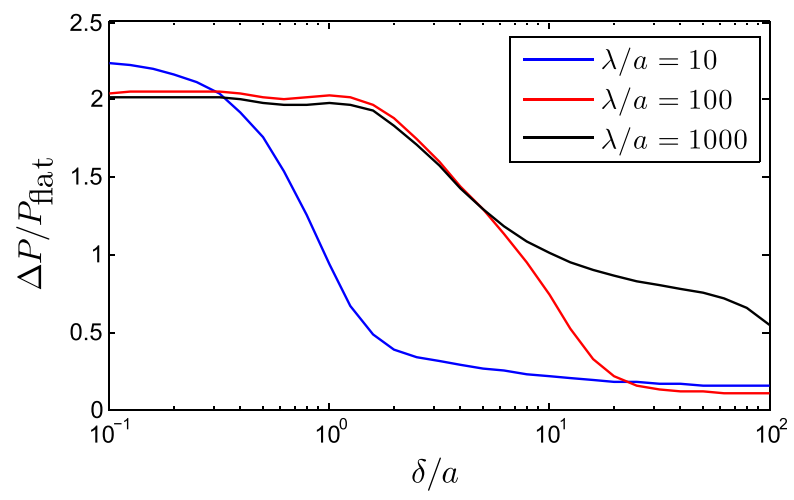

(a) $\mathbf{E}_{0}$

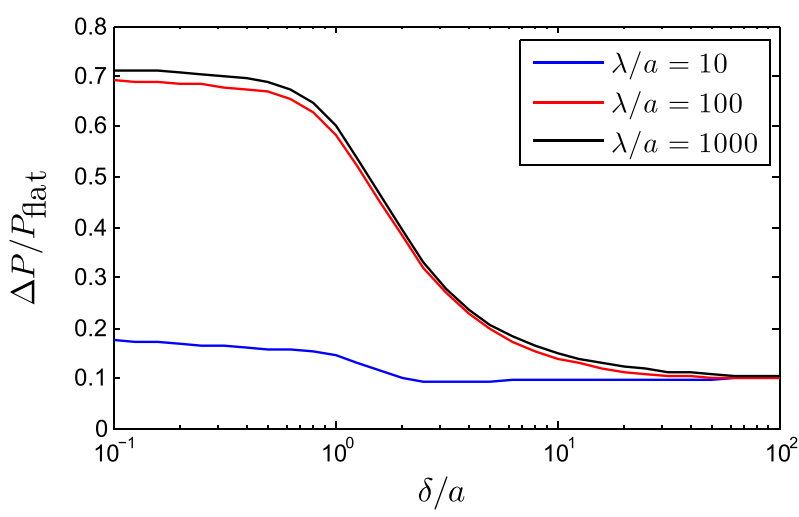

(b) $\mathbf{H}_{01}$

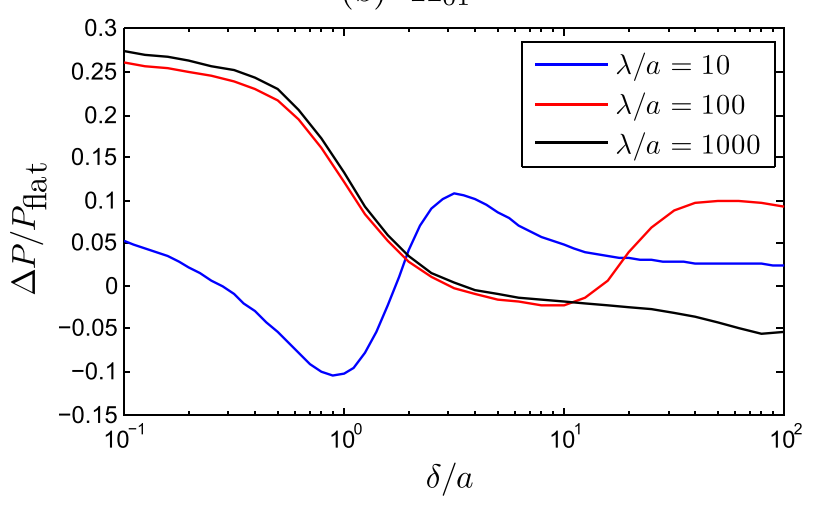

(c) $\mathbf{H}_{02}$

FIG. 5. $\Delta P / P_{\text {flat }}$ for various values of $\delta / a$ computed using HIEM for the case of the bump on the plane of finite conductivity. 


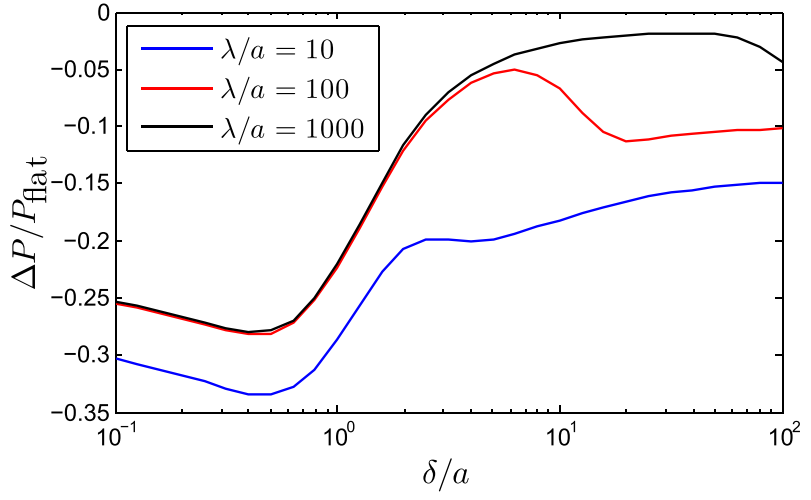

(a) $\mathbf{E}_{0}$

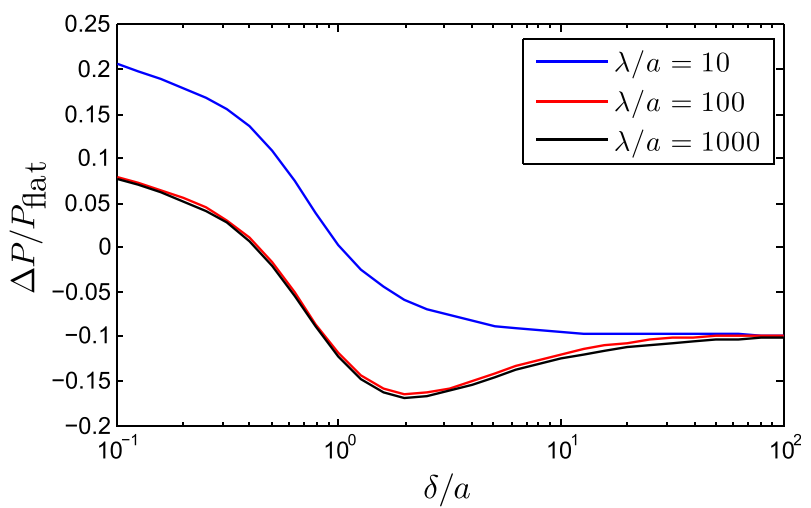

(b) $\mathbf{H}_{01}$

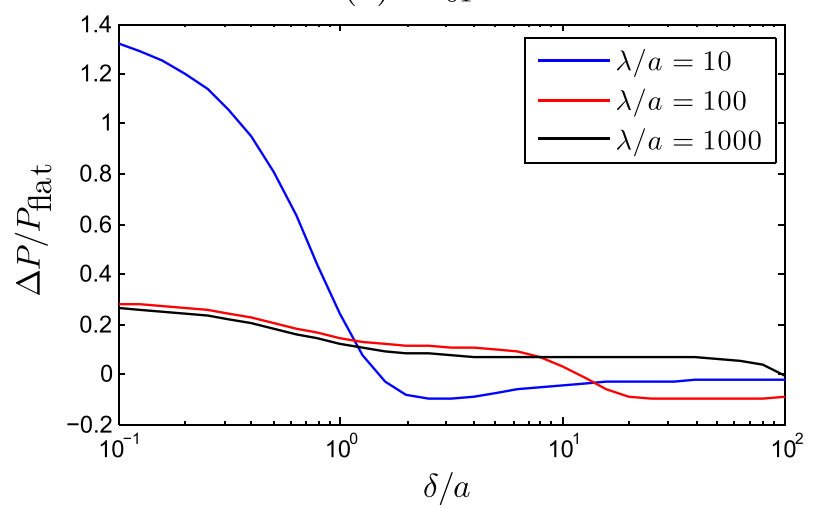

(c) $\mathbf{H}_{02}$

FIG. 6. $\Delta P / P_{\text {flat }}$ for various values of the skin depth $\delta / a$ computed using HIEM for the case of the trench on the plane of finite conductivity.

and minimum values of $|\mathbf{E}|$ inside the pristine conducting plane (in absence of the defect). In order to generate the plots for the cases $\mathbf{E}_{0}$ and $\mathbf{H}_{02}$, the electric field is computed by evaluating the curl of the magnetic field by means of second-order finite differences on a polar grid around the surface of the defect and on a Cartesian grid everywhere else.

Interestingly, from Figs. 5(c) and 6(c) we observe that for the $\mathbf{H}_{02}$ incident field and for large wavelength values $(\lambda / a \gg 10)$ the quotient $\Delta P / P_{\text {flat }}$ seems to approach the limit $\Delta L / L_{\text {flat }}=\left(L_{\text {rough }}-L_{\text {flat })} / L_{\text {flat }}=(\pi-2) / 4=0.2854\right.$ as $\delta \rightarrow 0$, where $L_{\text {rough }}=a(\pi+2)$ and $L_{\text {flat }}=4 a$ denote the length of the curve representing the boundary of the conducting surface on which the skin depth effect takes place in presence and absence of the defect respectively. In view of

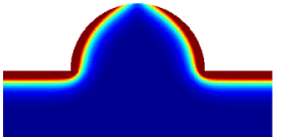

(a)

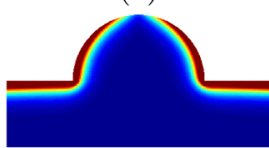

(d)

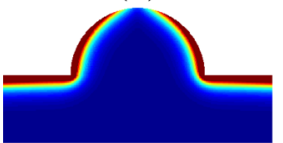

(g) (b)

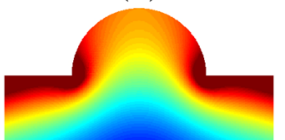

(e)

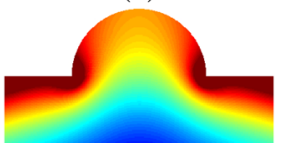

(h)

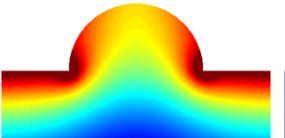

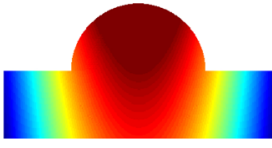

(c)

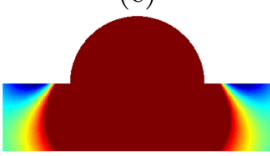

(f)

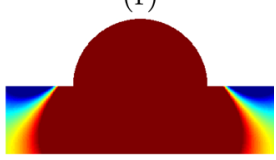

(i)
FIG. 7. Magnitude of the total electric field $|\mathbf{E}|$ for the case $\mathbf{E}_{0}$ in a region around the bump on the conducting plane of the same electric properties for $\delta / a=0.1$ (1st column), $\delta / a=1$ (2nd column), $\delta / a=10$ (3rd column), $\lambda / a=10$ (1st row), $\lambda / a=100$ (2nd row), and $\lambda / a=1000$ (3rd row).

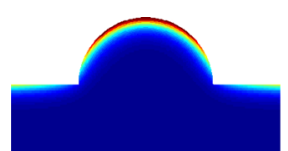

(a)

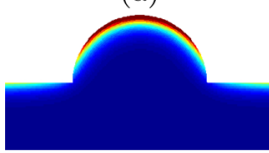

(d)

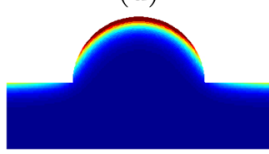

(g)

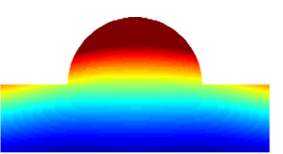

(b)

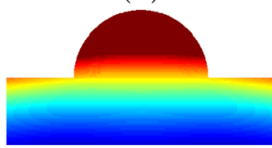

(e)

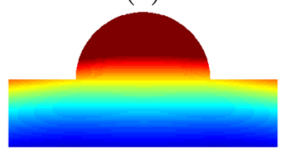

(h)

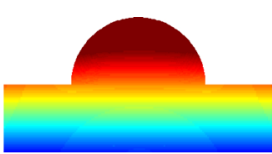

(c)

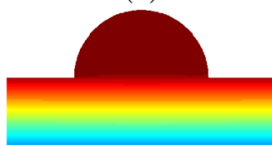

(f)

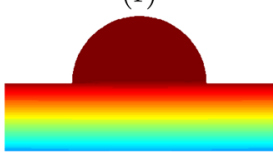

(i)
FIG. 8. Magnitude of the total electric field $|\mathbf{E}|$ for the incident field $\mathbf{H}_{01}$ in a region around the bump on the conducting plane of the same electric properties for $\delta / a=0.1$ ( $1 \mathrm{st}$ column), $\delta / a=1$ ( 2 nd column), $\delta / a=10$ ( 3 rd column), $\lambda / a=10$ (1st row), $\lambda / a=100$ (2nd row), and $\lambda / a=1000$ (3rd row).

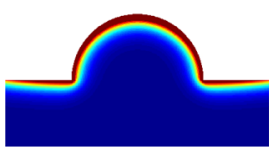

(a)

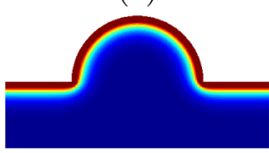

(d)

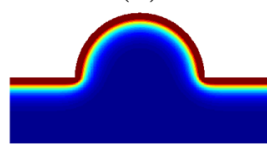

(g)

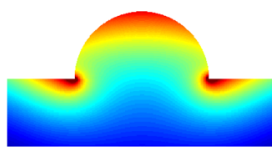

(b)

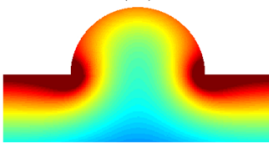

(e)

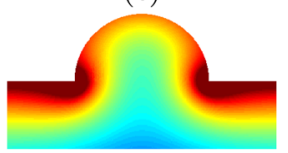

(h)

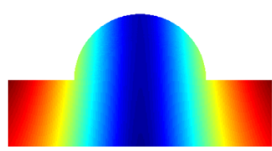

(c)

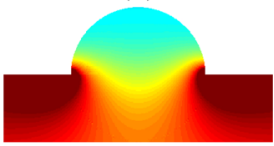

(f)

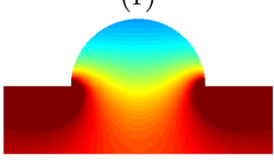

(i)
FIG. 9. Magnitude of the total electric field $|\mathbf{E}|$ for the incident field $\mathbf{H}_{02}$ in a region around the bump on the conducting plane of the same electric properties for $\delta / a=0.1$ ( 1 st column), $\delta / a=1$ ( 2 nd column), $\delta / a=10$ ( $3 \mathrm{rd}$ column), $\lambda / a=10$ (1st row), $\lambda / a=100$ (2nd row), and $\lambda / a=1000$ (3rd row). 


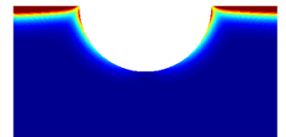

(a)

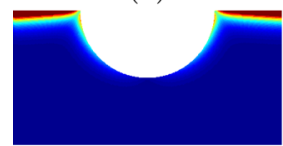

(d)

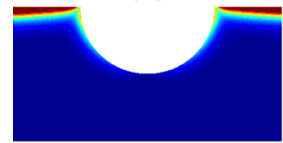

(g)

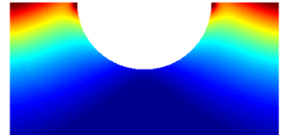

(b)

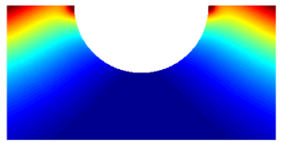

(e)

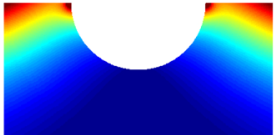

(h)

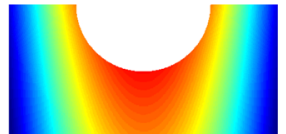

(c)

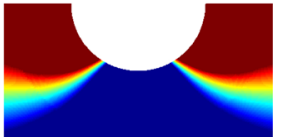

(f)

(i)

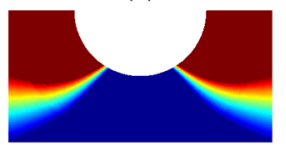

FIG. 10. Magnitude of the total electric field $|\mathbf{E}|$ for the incident field $\mathbf{E}_{0}$ in a region around the trench on the conducting plane of the same electric properties for $\delta / a=0.1$ ( 1 st column), $\delta / a=1$ ( 2 nd column), $\delta / a=10$ (3rd column), $\lambda / a=10$ (1st row), $\lambda / a=100$ (2nd row), and $\lambda / a=1000$ (3rd row).

Figs. 9(d), 9(g), 12(d), and 12(g), this phenomenon may be explained by the fact that in these cases the skin depth effect produces an uniform boundary layer near the boundary of the conducting surface. Note that if a different value of $L_{\mathrm{flat}}$ is used, the numerical values of Eq. (8) would change, so would the curves in Figs. 5 and 6. The electric field patterns shown in Figs. 7-12 remain unchanged, of course.

Surprisingly, in Fig. 6 we observe that, for some values of $\delta / a$, the electromagnetic absorption is actually reduced (not increased!) by the presence of the trench, specially in the cases $\mathbf{E}_{0}$ and $\mathbf{H}_{01}$. As can be seen in the first two columns in Figs. 10 and 11, this may be explained by the fact that, for the cases $\mathbf{E}_{0}$ and $\mathbf{H}_{01}$, dissipation around the surface of the trench is reduced.

We mention some salient features in the electric field patterns presented in the six $3 \times 3$ figure arrays labeled as Figs. 7-12. We first note that four out of the six (c) entries in these arrays, namely, Figs. 7(c), 9(c), 10(c), and 12(c), are qualitatively different from all other entries in the respective arrays. We conjecture that these striking differences relate to corresponding marked differences in the magnitude ratios

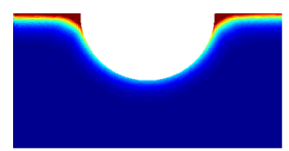

(a)

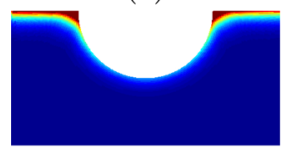

(d)

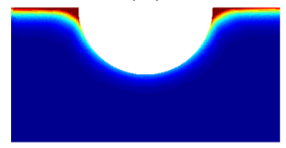

$(\mathrm{g})$

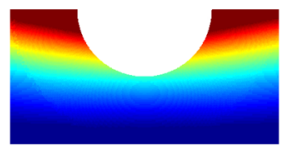

(b)

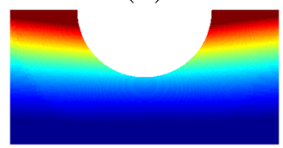

(e)

(h)

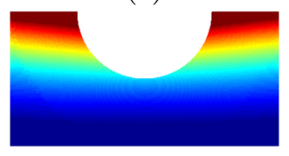

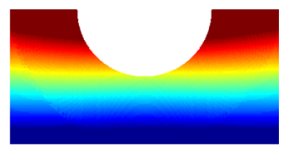

(c)

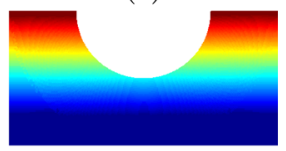

(f)

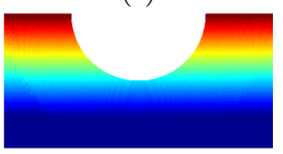

(i)
FIG. 11. Magnitude of the total electric field $|\mathbf{E}|$ for the incident field $\mathbf{H}_{01}$ in a region around the trench on the conducting plane of the same electric properties for $\delta / a=0.1$ ( 1 st column), $\delta / a=1$ (2nd column), $\delta / a=10$ (3rd column), $\lambda / a=10$ (1st row), $\lambda / a=100$ (2nd row), and $\lambda / a=1000$ (3rd row).
$R$ of the conduction current to the displacement current $\left(R=\sigma / \omega \epsilon_{0}=(\lambda / a)^{2} / 2(\pi \delta / a)^{2}\right)$ : the ratio $R$ equals 0.05 in all of the entries (c) in Figs. 7-12, but it is either equal to or much higher than 5 for all other entries. In particular, only for the (c) entries is the displacement current much larger than the conduction current; the opposite is true for the remaining entries. (Comments concerning Figs. 8(c) and 11 (c) are provided below in this section.)

Next, for the $\mathbf{E}_{0}$ case, say in Figs. 7(a) and 7(b), the RF magnetic field behaves like $H_{z} \sim \sin \left(k_{2} x\right)$ on the flat surface in the absence of the bump. While $H_{z}$ is zero near $x=0$, it becomes quite high near $x= \pm a$ in Figs. 7(a) and 7(b). Since the conduction current dominates in Figs. 7(a) and 7(b), the RF heating is dominated by the ohmic current of the inductive electric field that is caused by the RF magnetic field. ${ }^{36,37}$ This explains the electric field patterns shown in Figs. 7(a) and 7(b), and qualitatively Figs. 7(d), 7(e), 7(g), and 7(h). Figures 7(b), 7(e), 7(h), 7(f), and 7(i), may be readily understood in terms of increased skin depths. Similar explanations may be applied to Figs. 10(a), $10(\mathrm{~b}), 10(\mathrm{~d}), 10(\mathrm{e}), 10(\mathrm{~g})$, and $10(\mathrm{~h})$, bearing in mind that there is a reduction of the RF magnetic field at the trench (the RF electric field is also reduced there). Note that Figs. 10(f) and 10(i) are similar to Figs. 10(e) and 10(h), except that the skin depth of the former is 10 times larger than the latter.

For the first $\mathbf{H}_{02}$ case, Fig. 9(a), the RF magnetic field $H_{z}$ is dominant, and this is not affected by the bump. Thus, the heating is primarily due to the inductive electric field that is generated by $H_{z}$. This explains the electric field pattern in Fig. 9(a), and qualitatively Figs. 9(b), 9(d), 9(e), 9(g), and 9(h). We note that the severe current crowding shown in Figs. 9(f) and 9(i) is similar to the current crowding in thin films studied by Zhang et al. ${ }^{42}$ The current flow patterns in the trench cases shown in Figs. 12(a), 12(b), and 12(d)-12(i) are all qualitatively similar. Figure 12(c) has a different appearance because the displacement current dominates.

Finally, for the first $\mathbf{H}_{01}$ case, Fig. 8(a), the RF magnetic field $H_{x}$ is dominant. This "ambient" $H_{x}$, in the presence of a conducting bump, experiences a magnetic field enhancement at the bump's apex. This is why superconducting cavity

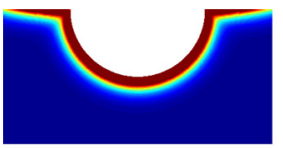

(a)

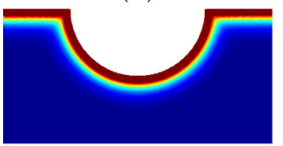

(d)

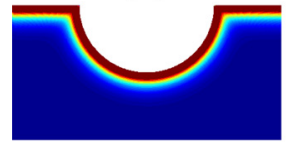

$(\mathrm{g})$

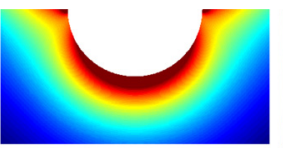

(b)

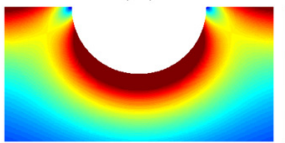

(e)

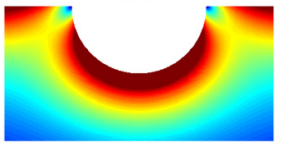

(h)

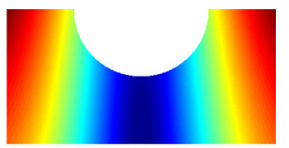

(c)

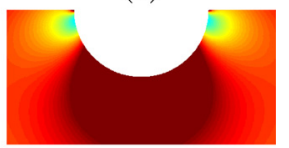

(f)

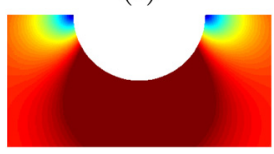

(i)
FIG. 12. Magnitude of the total electric field $|\mathbf{E}|$ for the incident field $\mathbf{H}_{02}$ in a region around the trench on the conducting plane of the same electric properties for $\delta / a=0.1$ (1st column), $\delta / a=1$ (2nd column), $\delta / a=10$ (3rd column), $\lambda / a=10$ (1st row), $\lambda / a=100$ (2nd row), and $\lambda / a=1000$ (3rd row). 
surface must be made very flat, otherwise the increase of magnetic field by surface roughness leads to quenching of a superconducting cavity. $2,7,9$ This is also why there would be a higher inductive heating at the apex of the bump in Figs. 8(a), $8(\mathrm{~d})$, and $8(\mathrm{~g})$. The remaining figures, 8(b), 8(c), 8(e), 8(f), $8(\mathrm{~h})$, and $8(\mathrm{i})$, may be qualitatively understood in terms of increased skin depths. On the contrary, in Fig. 11(a), for instance, the "ambient" magnetic field $H_{x}$ decreases at the valley of the trench. This explains the induction heating patterns shown in Figs. 11(a), 11(d), and 11(g). The remaining figures, 11(b), 11(c), 11(e), 11(f), 11(h), and 11(i), may be qualitatively understood in terms of increased skin depths in those parts.

\section{CONCLUSIONS}

This paper provides accurate evaluation of the electromagnetic field around a semi-cylindrical bump or trench on a locally flat conducting surface. Discussion of numerical results was restricted to cases in which (a) the size of the bump or trench is small compared with the wavelength of the radiation, and (b) the complex permittivity and permeability of the protrusion are constant. The latter constants may assume arbitrary values, however. The local RF electric and RF magnetic fields, $\mathbf{E}_{0}$ and $\mathbf{H}_{0}$ in the absence of the surface defect, may have arbitrary magnitudes and phases between them. The ohmic absorption by the bump and trench is calculated, and compared with the intrinsic ohmic absorption of an otherwise flat surface. The HIEM code ${ }^{32}$ was validated against analytic theory, and it was then applied to cases which defy analytic treatment. The complicated field patterns displayed in Figs. 7-12 are given relatively simple physical interpretations. Thus, our paper offers some new insights into the roles in ohmic heating of surface defects that are played by the individual components of the RF magnetic field and the RF electric field of the incident waves.

The HIEM code was subsequently applied to the regime where the wavelength is of the order of or shorter than the radius of the semi-cylindrical bump (or trench). This work will be published elsewhere. We also anticipate future applications that include interactions between defects (bumps and/ or trenches), irregular geometries, infinite arrays (periodic and random), and three-dimensional effects.

\section{ACKNOWLEDGMENTS}

This Caltech-Michigan collaboration was stimulated by discussions with Dr. Arje Nachman and Dr. John Luginsland of the Air Force Office of Scientific Research. The Caltech group was supported by AFOSR and NSF under Contract Nos. FA9550-11-1-0193 and DMS-1008631, respectively. The University of Michigan group was supported by AFOSR grants FA9550-09-1-0662, FA9550-14-1-0309, and L-3 Communication Electron Devices Division.

\section{APPENDIX: DERIVATION OF THE DAMPING RATES OF TE11, TM01 AND TE01 MODES DUE TO A SEMI-CYLINDRICAL BUMP}

In the absence of the bump, the semi-cylindrical cavity admits the TE11, TM01, and TE01 modes, as shown in Figs. 2(d)-2(f) respectively. We assume that the eigenfrequencies of these three modes all equal to $\omega$, by adjusting the radius of the cavity for these three modes. These modes will be damped if a lossy semi-cylindrical bump of radius $a$ is placed at the center $(z)$ axis of these cavities. Below we present the damping rates for $a / \lambda \ll 1$. We define $k_{1}=\omega\left(\varepsilon_{1} \mu_{1}\right)^{1 / 2}$, $k_{2}=\omega\left(\varepsilon_{2} \mu_{2}\right)^{1 / 2}, \quad \xi_{1}=k_{1} a, \quad \xi_{2}=k_{2} a, \quad \eta=k_{2} b, \quad$ and $Z_{1}=\sqrt{\left(\mu_{1} / \mu_{2}\right) /\left(\varepsilon_{1} / \varepsilon_{2}\right)}$. Note that $Z_{1}$ is simply the ratio of the characteristic impedance $(\mu / \varepsilon)^{1 / 2}$ of the two regions, the bump and its surrounding region (cf. Figs. 2(d)-2(f)).

\section{TE11 mode}

In the absence of the bump, the RF electric and magnetic fields are given by

$$
\begin{aligned}
H_{z} & =P J_{1}\left(k_{2} r\right) \cos \phi, \\
E_{\phi} & =\frac{k_{2}}{i \omega \varepsilon_{2}} P J_{1}^{\prime}\left(k_{2} r\right) \cos \phi, \\
E_{r} & =\frac{1}{i \omega \varepsilon_{2}} \frac{P}{r} J_{1}\left(k_{2} r\right) \sin \phi,
\end{aligned}
$$

where $P$ is a constant determined by the field strength, $J_{1}$ is the Bessel function of order 1 , the prime represents the derivative with respect to the argument, and the radius of the cavity $b_{E_{0}}$ satisfies

$$
\omega b_{E_{0}} \sqrt{\mu_{2} \varepsilon_{2}}=2 \pi b_{E_{0}} / \lambda=1.84118,
$$

in order that the eigenfrequency is $\omega$ for the TE11 mode. The presence of the bump of a small radius $\left(a \ll b_{E_{0}}\right)$ will perturb the eigenfrequency, yielding a damping rate $\gamma_{E_{0}}$ given by

$$
\begin{aligned}
\frac{\gamma_{E_{0}}}{\omega}= & -\frac{Y_{1}^{\prime}\left(\eta_{E_{0}}\right)}{\eta_{E_{0}} J_{1}^{\prime \prime}\left(\eta_{E_{0}}\right)} \frac{J_{1}\left(\xi_{2}\right)}{Y_{1}\left(\xi_{2}\right)}\left[B\left(\xi_{2}\right)-A\left(\xi_{2}\right)\right] \\
& \times \operatorname{Im}\left[\frac{1}{Z_{1} A\left(\xi_{1}\right)-B\left(\xi_{2}\right)}\right],
\end{aligned}
$$

where $\eta_{E_{0}}=1.84118, A(x)=J_{1}^{\prime}(x) / J_{1}(x), B(x)=Y_{1}^{\prime}(x) / Y_{1}(x)$. Equation (A2) has the following asymptotic dependence in the different ranges of $\delta / a$ :

$$
\frac{\gamma_{E_{0}}}{\omega} \cong\left\{\begin{array}{l}
4 \pi^{5} \alpha\left(\frac{a}{\lambda}\right)^{2} \sqrt{\frac{\mu_{1}}{\mu_{2}}}\left(\frac{a \delta}{\lambda^{2}}\right), \quad \sqrt{\frac{\mu_{2}}{\mu_{1}}} \frac{\delta}{a} \ll 1, \\
4 \pi^{5} \alpha\left(\frac{a \delta}{\lambda^{2}}\right)^{2}, 1 \ll \sqrt{\frac{\mu_{2}}{\mu_{1}}} \frac{\delta}{a} \ll \frac{1}{\sqrt{2} \pi} \sqrt{\frac{\varepsilon_{2} \mu_{2}}{\varepsilon_{1 r} \mu_{1}}} \frac{\lambda}{a}, \\
\pi \alpha\left(\frac{a}{\delta}\right)^{2}\left(\frac{\varepsilon_{2}}{\varepsilon_{1 r}+\varepsilon_{2}}\right)^{2}, \frac{1}{\sqrt{2} \pi} \sqrt{\frac{\varepsilon_{2} \mu_{2}}{\varepsilon_{1 r} \mu_{1}}} \frac{\lambda}{a} \ll \sqrt{\frac{\mu_{2}}{\mu_{1}}} \frac{\delta}{a},
\end{array}\right.
$$

where $\alpha=0.7868$. The derivation of Eqs. (A2) and (A3) follows Ref. 5. By comparing Eqs. (A2) and (A3), the exact solution obtained from the Fourier-Bessel expansion and the HIEM simulations are shown in Fig. 4(a). 


\section{TM01 mode}

In absence of the bump, the RF electric and magnetic fields are given by

$$
\begin{aligned}
E_{z} & =C J_{1}\left(k_{2} r\right) \cos \phi, \\
H_{\phi} & =-\frac{k_{2}}{i \omega \mu_{2}} C J_{1}^{\prime}\left(k_{2} r\right) \cos \phi, \\
H_{r} & =-\frac{1}{i \omega \mu_{2}} \frac{C}{r} J_{1}\left(k_{2} r\right) \sin \phi,
\end{aligned}
$$

where $C$ is a constant determined by the field strength and the radius of the cavity $b_{H_{01}}$ satisfies

$$
\omega b_{H_{01}} \sqrt{\mu_{2} \varepsilon_{2}}=2 \pi b_{H_{01}} / \lambda=3.83171,
$$

in order that the eigenfrequency is $\omega$ for the TM01 mode. The presence of a bump of a small radius $\left(a \ll b_{H_{01}}\right)$ will perturb the eigenfrequency, yielding a damping rate $\gamma_{H_{01}}$ given by

$$
\begin{aligned}
\frac{\gamma_{H_{01}}}{\omega}= & -\frac{Y_{1}\left(\eta_{H_{01}}\right)}{\eta_{H_{01}} J_{1}^{\prime}\left(\eta_{H_{01}}\right)} \frac{J_{1}^{\prime}\left(\xi_{2}\right)}{Y_{1}^{\prime} \xi_{2}}\left[\frac{1}{B\left(\xi_{2}\right)}-\frac{1}{A\left(\xi_{2}\right)}\right] \\
& \times \operatorname{Im}\left[\frac{1}{Z_{1} / A\left(\xi_{1}\right)-1 / B\left(\xi_{2}\right)}\right],
\end{aligned}
$$

where $\quad \eta_{H_{01}}=3.83171, A(x)=J_{1}^{\prime}(x) / J_{1}(x), B(x)=Y_{1}^{\prime}(x) /$ $Y_{1}(x)$. Equation (A5) has the following asymptotic dependence in different ranges of $\delta / a$ :

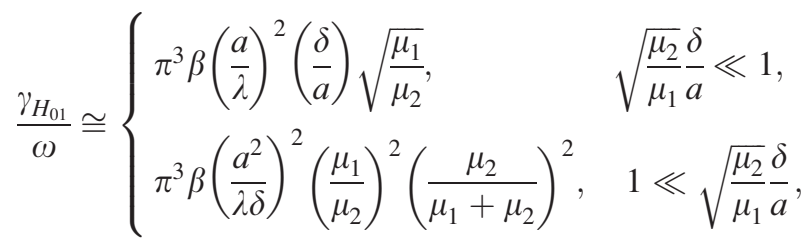

where $\beta=0.2673$. The derivation of Eqs. (A5) and (A6) follows Ref. 5. By comparing Eqs. (A5) and (A6), the exact solution obtained from the Fourier-Bessel expansion and the HIEM simulations are shown in Fig. 4(b).

\section{TE01 mode}

In absence of the bump, the RF electric and magnetic fields are given by

$$
\begin{aligned}
H_{z} & =D J_{0}\left(k_{2} r\right), \\
E_{\phi} & =\frac{k_{2}}{i \omega \varepsilon_{2}} D J_{0}^{\prime}\left(k_{2} r\right), \\
E_{r} & =0,
\end{aligned}
$$

where $D$ is a constant determined by the field strength and the radius of the cavity $b_{H_{02}}$ satisfies

$$
\omega b_{H_{02}} \sqrt{\mu_{2} \varepsilon_{2}}=2 \pi b_{H_{02}} / \lambda=3.83171,
$$

in order that the eigenfrequency is $\omega$ for the TE01 mode. The presence of a bump of a small radius $\left(a \ll b_{H_{02}}\right.$ ) will perturb the eigenfrequency, yielding a damping rate $\gamma_{H_{02}}$ given by

$$
\begin{aligned}
\frac{\gamma_{H_{02}}}{\omega}= & -\frac{Y_{1}\left(\eta_{H_{02}}\right)}{\eta_{H_{02}} J_{1}^{\prime}\left(\eta_{H_{02}}\right)} \frac{J_{0}\left(\xi_{2}\right)}{Y_{0}\left(\xi_{2}\right)}\left[B\left(\xi_{2}\right)-A\left(\xi_{2}\right)\right] \\
& \times \operatorname{Im}\left[\frac{1}{Z_{1} A\left(\xi_{1}\right)-B\left(\xi_{2}\right)}\right],
\end{aligned}
$$

where $\quad \eta_{H_{02}}=3.83171, A(x)=J_{1}(x) / J_{0}(x), B(x)=Y_{1}(x) /$ $Y_{0}(x)$. Equation (A8) has the following asymptotic dependence in different ranges of $\delta / a$ :

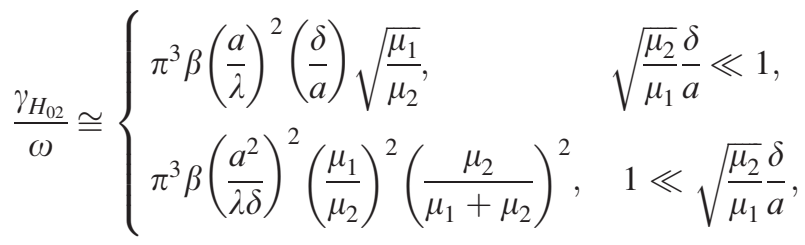

where $\beta=0.2673$. The derivation of Eqs. (A8) and (A9) follows Ref. 5. By comparing Eqs. (A8) and (A9), the exact solution obtained from the Fourier-Bessel expansion and the HIEM simulations are shown in Fig. 4(c).

${ }^{1}$ S. P. Morgan, J. Appl. Phys. 20, 352-362 (1949).

${ }^{2}$ H. Padamsee, Supercond. Sci. Technol. 14, R28 (2001).

${ }^{3}$ D. P. Pritzkau and R. H. Siemann, Phys. Rev. Spec. Top. Accel. Beams 5, 112002 (2002).

${ }^{4}$ T. Sun, B. Yao, A. P. Warren, K. Barmak, M. F. Toney, R. E. Peale, and K. R. Coffey, Phys. Rev. B 79, 041402 (2009).

${ }^{5}$ P. Zhang, Y. Y. Lau, and R. M. Gilgenbach, J. Appl. Phys. 105, 114908 (2009).

${ }^{6}$ B. B. Yang, S. L. Katz, K. J. Willis, M. J. Weber, I. Knezevic, S. C. Hagness, and J. H. Booske, IEEE Trans. Terahertz Sci. Technol. 2, 449-459 (2012)

${ }^{7}$ V. Shemelin and H. Padamsee, "Magnetic field enhancement at pits and bumps on the surface of superconducting cavities," TTC-Report 7, 2008.

${ }^{8}$ J. H. Booske, Phys. Plasmas 15, 055502 (2008).

${ }^{9}$ Y. Iwashita, Y. Tajima, and H. Hayano, Phys. Rev. Spec. Top. Accel. Beams 11, 093501 (2008).

${ }^{10}$ G. P. Scheitrum, "Microfabricated MVEDs," in Modern Microwave and Millimeter Wave Power Electronics, edited by R. J. Barker, N. C. Luhmann, J. H. Booske, and G. S. Nusinovich (IEEE Press, Piscataway, NJ, 2005), pp. 343-391.

${ }^{11}$ V. V. Bobkov, J.-M. Noterdaeme, and R. Wilhelm, Surf. Coat. Technol. 200, 822-826 (2005).

${ }^{12}$ H. Pandit, D. Shi, N. Hari Babu, X. Chaud, D. Cardwell, P. He, D. Isfort, R. Tournier, D. Mast, and A. M. Ferendeci, Physica C 425, 44-51 (2005).

${ }^{13}$ E. Collings, M. Sumption, and T. Tajima, Supercond. Sci. Technol. 17, S595 (2004).

${ }^{14}$ M. Lancaster, Passive Microwave Device Applications of High Temperature Superconductors (Cambridge University Press, New York, 1997).

${ }^{15}$ Z. Zhai, C. Kusko, N. Hakim, S. Sridhar, A. Revcolevschi, and A. Vietkine, Rev. Sci. Instrum. 71, 3151-3160 (2000).

${ }^{16} \mathrm{~L}$. Tsang, X. Gu, and H. Braunisch, IEEE Microwave Wireless Compon. 16, 221-223 (2006).

${ }^{17}$ A. F. Horn III, J. W. Reynolds, P. A. LaFrance, and J. C. Rautio, "Effect of conductor profile on the insertion loss, phase constant, and dispersion in thin high frequency transmission lines," DesignCon 2010 Proceedings, Santa Clara, CA, 2010.

${ }^{18}$ M. V. Lukic and D. S. Filipovic, IEEE Trans. Microwave Theory Tech. 55, 518-525 (2007).

${ }^{19}$ P. Zhang and Y. Y. Lau, J. Appl. Phys. 108, 044914 (2010).

${ }^{20} \mathrm{P}$. Zhang, "Effects of surface roughness on electrical contact, RF heating and field enhancement," Ph.D. thesis (The University of Michigan, Ann Arbor, 2012).

${ }^{21}$ R. Miller, Y. Y. Lau, and J. H. Booske, Appl. Phys. Lett. 91, 074105 (2007).

${ }^{22}$ K. L. Jensen, Y. Y. Lau, D. Feldman, and P. O’Shea, Phys. Rev. Spec. Top. Accel. Beams 11, 081001 (2008). 
${ }^{23}$ T. A. de Assis, F. Borondo, R. Benito, and R. Andrade, Phys. Rev. B 78 235427 (2008).

${ }^{24}$ A. A. Maradudin and D. L. Mills, Phys. Rev. B 11, 1392-1415 (1975).

${ }^{25}$ A. A. Maradudin, D. L. Mills, and R. F. Wallis, "Theory of Rayleigh wave scattering by mass defects and surface roughness," in Phonon Scattering in Solids, edited by L. J. Challis, V. W. Rampton, and A. F. G. Wyatt (Springer, 1976), pp. 55-57.

${ }^{26}$ S. Groiss, I. Bardi, O. Biro, K. Preis, and K. Richter, IEEE Trans. Magn. 32, 894-897 (1996).

${ }^{27} \mathrm{See}$ http://www.ansoft.com for the HFSS codes.

${ }^{28}$ C. L. Holloway and E. F. Kuester, IEEE Trans. Microwave Theory Tech. 48, 1601-1610 (2000).

${ }^{29}$ A. Matsushima and K. Nakata, Electron. Commun. Jpn., Part 2 89, 1-10 (2006).

${ }^{30}$ M. A. Biot, J. Appl. Phys. 28, 1455-1463 (1957).

${ }^{31}$ J. Wait, IRE Trans. Antennas Propag. 7, 154-162 (1959).

${ }^{32}$ C. Pérez-Arancibia and O. P. Bruno, J. Opt. Soc. Am. A 31, 1738-1746 (2014).

${ }^{33}$ A. Taflove and S. C. Hagness, Computational Electrodynamics: The Finite-Difference Time-Domain Method (Artech House, 2005).
${ }^{34} \mathrm{~J}$. Jin, The Finite Element Method in Electromagnetics (John Wiley \& Sons, 2014).

${ }^{35}$ J. D. Jackson, Classical Electrodynamics (John Wiley \& Sons, New York, 1998).

${ }^{36}$ H. Bosman, W. Tang, Y. Y. Lau, and R. M. Gilgenbach, Appl. Phys. Lett. 85, 3319-3321 (2004).

${ }^{37}$ W. Tang, H. Bosman, Y. Y. Lau, and R. M. Gilgenbach, J. Appl. Phys. 97, 114915 (2005).

${ }^{38}$ S. Ramo, J. R. Whinnery, and T. V. Duzer, Fields and Waves in Communication Electronics (Wiley, New York, 1994).

${ }^{39}$ M. K. Hinders and A. D. Yaghjian, IEEE Microwave Guided Wave Lett. 1, 239-242 (1991).

${ }^{40}$ J. Cheng, R. Roy, and D. Agrawal, J. Mater. Sci. Lett. 20, 1561-1563 (2001).

${ }^{41}$ L. Landau, L. Pitaevskii, and E. Lifshitz, Electrodynamics of Continuous Media, Course of Theoretical Physics Vol. 8 (Butterworth-Heinemann, 1984).

${ }^{42}$ P. Zhang, D. Hung, and Y. Y. Lau, J. Phys. D: Appl. Phys. 46, 065502 (2013); Corrigendum 46, 209501 (2013). 